\title{
Myxoinflammatory Fibroblastic Sarcoma
}

National Cancer Institute

\section{Source}

National Cancer Institute. Myxoinflammatory Fibroblastic Sarcoma. NCI Thesaurus. Code C49025.

A low grade, locally aggressive, fibroblastic neoplasm that occurs primarily in the distal extremities. It is characterized by the presence of spindle-shaped fibroblasts,

multivacuolated lipoblast-like cells, bizarre gang lion-like cells with inclusion-like nuclei, myxoid stroma formation, and a mixture of acute and chronic inflammatory cells. Distant metastases are very rare. 